\title{
High-spin studies and nuclear structure in three semi-magic regions of the chart: High-seniority states in $\mathrm{Sn}$ isotopes
}

\author{
Alain Astier \\ CSNSM, IN2P3-CNRS and Université Paris-Sud, 91405 Orsay, France
}

\begin{abstract}
Two fusion-fission experiments have been performed and studied with the Euroball Ge array: ${ }^{12} \mathrm{C}+{ }^{238} \mathrm{U}$ at $90 \mathrm{MeV}$ bombarding energy, and ${ }^{18} \mathrm{O}+{ }^{208} \mathrm{~Pb}$ at $85 \mathrm{MeV}$. Among the lot of new information extracted during the last decade, the latest results discussed here are the discovery of the high-spin states of ${ }^{119-126} \mathrm{Sn}$. The maximum value of angular momentum available in the $\nu \mathrm{h}_{11 / 2}$ shell, i.e. for mid-occupation and the breaking of the three neutron pairs (seniority $\mathrm{v}=6$ ), has been identified in several tin isotopes. It is the first time that such high-seniority states are established in spherical nuclei.
\end{abstract}

\section{Introduction}

In addition to deep inelastic collisions, the fission process (either spontaneous or induced by heavy ions) is a very convenient tool to produce a large number of neutron-rich isotopes in a single experiment. When combined to a powerful gamma detection system, it then offers the possibility to cover a large variety of nuclear structure phenomena, from the study of the high-spin states of fission products. Two fusion-fission have been performed and extensively studied this last decade. A lot of new results have been extracted, about the high-spin states of many fission fragments. Our new results concerning the tin isotopes are discussed in this paper.

The identification of states involving many identical nucleons in the same orbit $j$, i.e. states with the $j^{n}$ configuration, is a straightforward application of the nuclear shell model. However, pure $j^{n}$ configurations occur in a very few nuclei, since an orbit $j$ is rarely bounded by two gaps in energy, and the closeness of several orbits leads to configuration mixings. Nevertheless, some of the features due to the seniority are found to survive. For instance there are in semi-magic nuclei fairly constant spacings between the $0^{+}$ground state and some $v=2$ states, even though large changes in configuration mixings occur. The Sn nuclei provide a typical example; the energies of their first two states $\left(2^{+}\right.$and $4^{+}$in the even isotopes) do not vary much across the major shell $(54<\mathrm{N}<80)$, while the neutron orbits evolve from $\left(\mathrm{d}_{5 / 2}, \mathrm{~g}_{7 / 2}\right)$ for $\mathrm{N}<64$, to $\left(\mathrm{s}_{1 / 2}, \mathrm{~h}_{11 / 2}, \mathrm{~d}_{3 / 2}\right)$ for $\mathrm{N}>64$.

While there are numerous examples of states with seniority $v=2$ in the literature, only a few states with $v=4$ are known: The $10^{+}$and $12^{+}$of the $\pi\left(\mathrm{g}_{9 / 2}\right)^{4}$ configuration have been identified in two $N=50$ isotones, ${ }^{94} \mathrm{Ru}$ and ${ }^{96} \mathrm{Pd}$, and the $16^{+}$state of the $\pi\left(\mathrm{h}_{11 / 2}\right)^{4}$ configuration in the $\mathrm{N}=82$ nucleus,

\footnotetext{
ae-mail: Alain. Astier@csnsm.in2p3.fr
}

This is an Open Access article distributed under the terms of the Creative Commons Attribution License 2.0, which permits unrestricted use, distribution, and reproduction in any medium, provided the original work is properly cited. 
${ }^{150} \operatorname{Er}[1]$. The Sn isotopic chain is a good laboratory to study the case of $v\left(\mathrm{~h}_{11 / 2}\right)^{\mathrm{n}}$, particularly the highest spin states expected for $\mathrm{n}=4,5$ and 6. Indeed, the high-spin states of the $\mathrm{Sn}$ isotopes do contain a large $v\left(\mathrm{~h}_{11 / 2}\right)^{\mathrm{n}}$ component, since the two other valence orbits, $\nu \mathrm{s}_{1 / 2}$ and $\nu \mathrm{d}_{3 / 2}$, cannot afford large spin values. However, such a work is restricted to $\mathrm{N} \geq 70$ since the yrast states of the lightest $\mathrm{Sn}$ isotopes are dominated by a collective band coming from two-particle-two-hole excitations across the $\mathrm{Z}=50$ gap; and the states coming from the $v\left(\mathrm{~h}_{11 / 2}\right)^{\mathrm{n}}$ cannot be identified.

For that purpose their yrast states have to be populated with enough intensity to be measured. Because of the lack of suitable stable projectile-target combinations, high-spin states of heavy $\mathrm{Sn}$ isotopes cannot be populated by fusion-evaporation reactions. Thus up to now, the medium spin states of the ${ }^{120-126} \mathrm{Sn}$ isotopes were only measured up to the spin $\mathrm{I}^{\pi}=10^{+}$for the even mass and $\mathrm{I}^{\pi}=27 / 2^{-}$ for the odd mass, by using reactions induced by light ions, deep inelastic reactions, isomeric decays of long-lived states of Sn produced by fission of actinides, or $\beta$-decays of the high-spin long-lived states of heavy indium nuclei [2-8]. The decay of a new isomeric state in ${ }^{128} \mathrm{Sn}$ populated in the fragmentation of ${ }^{136} \mathrm{Xe}$ has been recently reported [9] which has been proposed to be the $15^{-}$state expected from the $\left(v \mathrm{~h}_{11 / 2}\right)^{-3}\left(\nu \mathrm{d}_{3 / 2}\right)^{-1}$ configuration.

For the studies presented in this paper, the ${ }^{119-126} \mathrm{Sn}$ isotopes have been produced as fragments of binary fission induced by heavy ions. New results have been found in these nuclei. Among them, isomeric states lying around $4.5 \mathrm{MeV}$ have been established in ${ }^{120,122,124,126} \mathrm{Sn}$ from the delayed coincidences between fission fragment detectors and the gamma array. All the observed states can be described in terms of broken neutron pairs occupying the $v \mathrm{~h}_{11 / 2}$ orbit. The maximum value of angular momentum available in this high- $j$ shell, i.e. for mid-occupation and the breaking of the three pairs, has been identified [10].

\section{Experimental conditions}

\subsection{Reaction and detection}

The ${ }^{12} \mathrm{C}+{ }^{238} \mathrm{U}$ reaction was performed at $90 \mathrm{MeV}$ incident energy. The beam was provided by the Legnaro XTU tandem accelerator. The $47 \mathrm{mg} / \mathrm{cm}^{2}$ target of ${ }^{238} \mathrm{U}$ was thick enough to stop the recoiling nuclei. The second reaction, ${ }^{18} \mathrm{O}+{ }^{208} \mathrm{~Pb}$ at $85 \mathrm{MeV}$ beam energy, was studied at the Vivitron accelerator of IReS (Strasbourg). The thickness of the target was $100 \mathrm{mg} / \mathrm{cm}^{2}$. In these two experiments, the $\gamma$ rays were detected with the Euroball array consisting of 71 Compton-suppressed Ge detectors [11]. The data were recorded in an event-by-event mode with the requirement that a minimum of five (three) unsuppressed Ge detectors fired in prompt coincidence (within a time window of $50 \mathrm{~ns}$ ) during the first (second) experiment. About $1.9 \times 10^{9}\left(4 \times 10^{9}\right)$ coincidence events (within a time window of $300 \mathrm{~ns}$ ) with a $\gamma$ multiplicity greater than or equal to three were registered. The offline analysis consisted of both multi-gated spectra and several three-dimensional "cubes" built and analysed with the Radware package [12].

To identify new isomeric states in fission fragments, we have performed another experiment using a fission fragment detector to trigger the Euroball array and isolate the delayed $\gamma$-ray cascades. The heavy-ion detector (SAPhIR: Saclay Aquitaine Photovoltaic cells for Isomer Research) is made of many photovoltaic cells which can be arranged in several geometries [13]. In the present work, it consisted of 32 photovoltaic modules lying in four rings around the target. We have used the ${ }^{12} \mathrm{C}+{ }^{238} \mathrm{U}$ reaction at $90 \mathrm{MeV}$ with a thin target, $0.14 \mathrm{mg} / \mathrm{cm}^{2}$. Fragments escaping from the target are stopped in the photovoltaic cells of SAPhIR. The detection of the two fragments in coincidence provides a clean signature of fission events. The Euroball time window was [50 ns $-1 \mu \mathrm{s}$ ], allowing detection of delayed $\gamma$ rays emitted during the de-excitation of isomeric states. Time spectra between fragments and $\gamma$ rays were analysed in order to measure the half-life of the isomeric levels discovered in the ${ }^{120,122,124} \mathrm{Sn}$ nuclei. 


\section{$2.2 \gamma-\gamma$ angular correlations}

In order to determine the spin values of excited states, the coincidence rates of two successive $\gamma$ transitions are analysed as a function of $\theta$, the average relative angle between the two fired detectors. The Euroball spectrometer had 28441 combinations of 2 crystals, out of which only $\sim 2000$ involved different values of relative angle within $2^{\circ}$. Therefore, in order to keep reasonable numbers of counts, all the angles have been gathered around three average relative angles: $22^{\circ}, 46^{\circ}$, and $75^{\circ}$. The coincidence rate is increasing between $0^{\circ}$ and $90^{\circ}$ for the dipole-quadrupole cascades, whereas it decreases for the quadrupole-quadrupole or dipole-dipole ones. More precisely, the angular correlation functions at the three angles of interest were calculated for several combinations of spin sequences, corresponding to typical multipole orders. In order to check the method, angular correlations of transitions belonging to the yrast cascades of the fission fragments having well-known multipole orders were analysed and the expected values were found in all cases.

When the statistics of our data are too low to perform such a measurement, the spin assignments are based upon (i) the already known spins of some states, (ii) the assumption that in yrast decays, spin values increase with the excitation energy, (iii) the possible existence of cross-over transitions, and (iv) the analogy with the level structures of the other isotopes.

\section{High-spin states in tin isotopes}

\subsection{Identification of new $\gamma$ ray cascades in Sn isotopes}

The identification of transitions depopulating high-spin levels which are completely unknown is based on the fact that prompt $\gamma$ rays emitted by complementary fragments are detected in coincidence $[14,15]$. For each reaction used in this work, we have studied the intensities of $\gamma$ rays emitted by many pairs of complementary fragments with known cascades to establish the relationship between their number of protons and neutrons. The sum of the proton numbers of complementary fragments has been found to be the atomic number of the compound nucleus, so that the ${ }_{50} \mathrm{Sn}$ isotopes are associated to the ${ }_{48} \mathrm{Cd}$ isotopes in the ${ }^{12} \mathrm{C}+{ }^{238} \mathrm{U}$ reaction, and to the ${ }_{40} \mathrm{Zr}$ isotopes in the ${ }^{18} \mathrm{O}+{ }^{208} \mathrm{~Pb}$ reaction. The distribution and the mean number of emitted neutrons depend slightly on the N/Z ratio of the investigated fragments and on the angular momentum of their excited states emitting the $\gamma$ rays.

Many new $\gamma$-ray cascades of the ${ }^{120-126}$ Sn nuclei have been identified using the distribution of masses of their partners, as explained below. It is worth noting that the use of two different reactions to produce the various $\mathrm{Sn}$ isotopes has turned out to be essential to disentangle the coincidence relationships which are often complicated by the existence of many doublets or triplets of transitions very close in energy.

The relative intensity of the lowest transitions in the new cascades identified in ${ }^{120-126} \mathrm{Sn}$ have been measured in the spectra in double coincidences with one new transition and one transition of a partner. As for the other transitions, we have used spectra in double coincidences with two transitions of the new cascades. A loss in intensity occurs when going through an isomeric state. Knowing that the time window was $300 \sim$ ns for the two experiments, such an effect has been taken into account for the halflives in the 100-300 ns range, observed in the present work.

\subsection{New level schemes of ${ }^{119-126} \mathrm{Sn}$}

Up to now, the high-spin level schemes of ${ }^{120-126} \mathrm{Sn}$ isotopes could only be established up to the longlived isomeric states, with $\mathrm{I}^{\pi}=10^{+}$or $27 / 2^{-}$at about $2-3 \mathrm{MeV}$ excitation energy, since it is difficult to register coincidence relationships between the transitions populating and depopulating states having such half-life values $\left(\mathrm{T}_{1 / 2}=1-62 \mu \mathrm{s}\right)$ using standard experimental apparatus. On the other hand, in the fusion-fission experiments, all the $\gamma$-ray cascades located above the long-lived isomeric states of 
the $\mathrm{Sn}$ isotopes are easily detected in prompt coincidences with those emitted by their complementary fragments. Three steps are necessary to carry out the search: i) Find all the $\gamma$ rays emitted in prompt coincidences with the transitions of the ${ }^{114-122} \mathrm{Cd}$ fragments in the first data set and the ${ }^{96-100} \mathrm{Zr}$ fragments in the second one, which do not belong to their respective level schemes; ii) Build all the $\gamma$-ray cascades using their own coincidences; iii) Assign each $\gamma$-ray cascade to one particular Sn isotope, from the distribution of masses of their partners.

For instance, in the spectrum gated by the $2^{+} \rightarrow 0^{+}$and $4^{+} \rightarrow 2^{+}$transitions of ${ }^{118} \mathrm{Cd}$, built from the data set of the ${ }^{12} \mathrm{C}+{ }^{238} \mathrm{U}$ reaction, a lot of new transitions are observed in the [950-1250]-keV energy range and can be assigned to ${ }^{120-125} \mathrm{Sn}$. In order to find the $\gamma$-ray cascades comprising these transitions, all the spectra in double coincidence with one transition of ${ }^{118} \mathrm{Cd}$ and one new transition have been precisely analysed. Then, in order to assign every $\gamma$-ray cascade to one particular $\mathrm{Sn}$ isotope, we have first analysed the relative intensities of $\gamma$ rays emitted by the partners in each spectrum in double coincidence with two transitions of the new cascades. Then we have analysed all these distributions of masses of the partners. The evolution of the $\mathrm{Cd}$ yields proves that the new cascades belong to different $\mathrm{Sn}$ isotopes. Similar results are obtained from the evolution of the $\mathrm{Zr}$ yields associated to each cascade, in the second experiment. Finally these relative distributions of $\mathrm{Cd} / \mathrm{Zr}$ masses were compared to wellknown pairs of complementary fragments and the new cascades were assigned to ${ }^{120,121,122,123,124,126} \mathrm{Sn}$.

Moreover, using the data from the SAPhIR experiment, we have found that several transitions belonging to these cascades are delayed, revealing the existence of isomeric states. The half-live of these new isomers have been measured in ${ }^{120,122,124} \mathrm{Sn}$. All the new information established in this work are gathered in the new level schemes presented in Ref. [10].

\section{Discussion}

The highest-spin states of the $\mathrm{Sn}$ isotopes with $\mathrm{N}>64$ do contain a large $v\left(\mathrm{~h}_{11 / 2}\right)^{\mathrm{n}}$ component, since the two low- $j$ orbits cannot afford large spin values. Therefore their study as a function of the neutron number gives us the opportunity to explore the main features of the $v\left(\mathrm{~h}_{11 / 2}\right)^{4,5,6}$ configurations. However such a work is restricted to $\mathrm{A} \geq 120$ since the yrast states of the lightest-A Sn isotopes are dominated by a collective band coming from $2 \mathrm{p}-2 \mathrm{~h}$ excitations across the $\mathrm{Z}=50$ gap, which hampers identifying states coming from the $v\left(\mathrm{~h}_{11 / 2}\right)^{\mathrm{n}}$ configurations.

From the systematics of the excitation energies of the highest-spin states in the ${ }^{120-128} \mathrm{Sn}$ even-A isotopes, reported in the left part of Fig. 1, one can see that the evolution of the positive-parity states is very smooth. Likewise, the energies of the negative-parity states display a very regular behaviour. This indicates that their main configurations are the same, whatever the number of neutrons. Being very close in energy for $69 \leq \mathrm{N} \leq 81$, the three neutron orbits $\left(\mathrm{s}_{1 / 2}, \mathrm{~h}_{11 / 2}, \mathrm{~d}_{3 / 2}\right)$ are gradually filled together. Then the occupation number of the $v\left(\mathrm{~h}_{11 / 2}\right)$ subshell does not change by two units from one even-A isotope to the next one.

Knowing the maximum values of angular momentum given by breaking up to three neutron pairs in the $v\left(\mathrm{~h}_{11 / 2}\right)$ orbit, it is tempting to assume that the positive-parity states have the $v\left(\mathrm{~h}_{11 / 2}\right)^{4}$ and $v\left(\mathrm{~h}_{11 / 2}\right)^{6}$ configurations, and the negative-parity states, the $v\left(\mathrm{~h}_{11 / 2}\right)^{3}\left(\mathrm{~d}_{3 / 2}\right)^{1}$ and $v\left(\mathrm{~h}_{11 / 2}\right)^{5}\left(\mathrm{~d}_{3 / 2}\right)^{1}$ configurations. Nevertheless we can notice immediately the peculiar behaviour of the $18^{+}$states: (i) the irregular variation of its excitation energy as a function of $\mathrm{A}$, and (ii) the low value of the $18^{+}-16^{+}$gap in energy. Thus the main configuration of the $18^{+}$states is likely not the $\mathrm{h}^{6}$ configuration.

In order to go further, we have performed shell-model calculations using the ANTOINE code [16], the calculation details being the same as those described in Ref. [17]. Since the present work mainly involves high-spin states, we have restricted the calculations to the excited states with spin values higher than $7^{-} / 10^{+}$for the even-A isotopes and $23 / 2^{+} / 27 / 2^{-}$for the odd-A ones. We only discuss in this paper the results for ${ }^{125} \mathrm{Sn}$ and ${ }^{126} \mathrm{Sn}$, as those of the lower-A isotopes are nearly the same. The comparison 

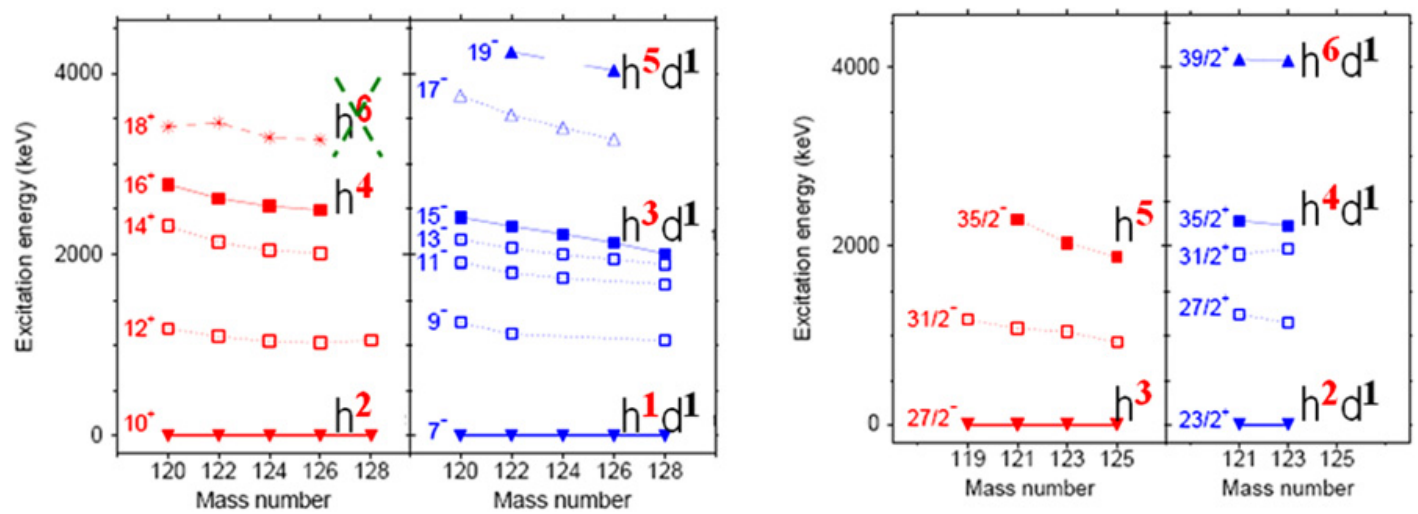

Figure 1. Experimental states observed in this work in the ${ }^{120-126} \mathrm{Sn}$ even isotopes (left) and ${ }^{119-125} \mathrm{Sn}$ odd isotopes (right). Only the states above the previously known long-lived isomers, taken as reference, are displayed. The states with configurations corresponding to a maximally-aligned spin, which involve successive pair breakings in the $v\left(\mathrm{~h}_{11 / 2}\right)$ orbit, are displayed with filled symbols.

of our results with those obtained for a pure $v\left(\mathrm{~h}_{11 / 2}\right)^{\mathrm{n}}$ configuration (performed in Ref. [18]\} is fruitful, since it shows the influence of the low- $j$ neutron orbits in the energies of these states. For instance, the $31 / 2^{-}$state is now located at mid-distance between the $35 / 2^{-}$and the $27 / 2^{-}$states. In the same manner, the $12^{+}$state is located at mid-distance between the $14^{+}$and the $10^{+}$states, while it is located nearer the $14^{+}$state in the first calculation. On the other hand, the states due to the complete alignment of the $\mathrm{h}_{11 / 2}$ momenta of the neutron belonging to the broken pairs are clearly identified. For instance, the main configuration of the $35 / 2^{+}$state of ${ }^{125} \mathrm{Sn}$ is $v\left(\mathrm{~s}_{1 / 2}\right)^{0}\left(\mathrm{~d}_{3 / 2}\right)^{3}\left(\mathrm{~h}_{11 / 2}\right)^{8}(61 \%)$, i.e. the breaking of two $v\left(\mathrm{~h}_{11 / 2}\right)$ pairs, and the main configuration of the $39 / 2^{+}$state is $v\left(\mathrm{~s}_{1 / 2}\right)^{2}\left(\mathrm{~d}_{3 / 2}\right)^{3}\left(\mathrm{~h}_{11 / 2}\right)^{6}(79 \%)$, i.e. the breaking of three $v\left(\mathrm{~h}_{11 / 2}\right)$ pairs. Results obtained for ${ }^{126} \mathrm{Sn}$ are very similar, except for the $18^{+}$yrast state. Its main configuration is $v\left(\mathrm{~s}_{1 / 2}\right)^{1}\left(\mathrm{~d}_{3 / 2}\right)^{3}\left(\mathrm{~h}_{11 / 2}\right)^{8}(59 \%)$, the $v\left(\mathrm{~s}_{1 / 2}\right)^{2}\left(\mathrm{~d}_{3 / 2}\right)^{4}\left(\mathrm{~h}_{11 / 2}\right)^{6}$ component being well weaker $(11 \%)$.

Conversely, the main component of the $18_{2}^{+}$state is $v\left(\mathrm{~s}_{1 / 2}\right)^{2}\left(\mathrm{~d}_{3 / 2}\right)^{4}\left(\mathrm{~h}_{11 / 2}\right)^{6}(79 \%)$, i.e. the breaking of three $v\left(\mathrm{~h}_{11 / 2}\right)$ pairs. Thus in ${ }^{126} \mathrm{Sn}$, the breaking of the third $v\left(\mathrm{~h}_{11 / 2}\right)$ pair, which only provides an angular momentum of $2 \hbar$, competes unfavourably with the breaking of a low- $j$ pair, which can also provide an angular momentum of $2 \hbar$, either from the $v\left(\mathrm{~s}_{1 / 2}\right)^{1}\left(\mathrm{~d}_{3 / 2}\right)^{3}$ configuration or from the $v\left(\mathrm{~d}_{3 / 2}\right)^{2}$ one. It is worth noting that such a process does not occur in the $39 / 2^{+}$state of ${ }^{125} \mathrm{Sn}$, because of the blocking of the $v\left(\mathrm{~d}_{3 / 2}\right)$ orbit by the odd neutron.

These theoretical results are in good agreement with the experimental ones; particularly the various distances in energy between successive states are well reproduced. First, the $14^{+}-12^{+}$distance is found to be close to the $12^{+}-10^{+}$one, as measured experimentally. Similar results are obtained for the $35 / 2^{-}-31 / 2^{-}$and $31 / 2^{-}-27 / 2^{-}$spacings which are well predicted. Secondly, the bunching of the three states with $\mathrm{I}^{\pi}=11^{-}, 13^{-}$, and $15^{-}$is well reproduced, as well as the large gap between the $15^{-}$and $17^{-}$states. Lastly, the peculiar behaviour of the experimental $18^{+}$states is likely due to the breaking of a low- $j$ neutron pair, which is expected to evolve as a function of A since the $v\left(\mathrm{~s}_{1 / 2}\right)$ orbit gets farther away from the neutron Fermi level as $\mathrm{N}$ is increasing.

In summary, we have identified states having the maximum value of angular momentum of either the $v\left(\mathrm{~h}_{11 / 2}\right)^{\mathrm{n}}$ configurations or the $v\left(\mathrm{~h}_{11 / 2}\right)^{\mathrm{n}}\left(\mathrm{d}_{3 / 2}\right)^{1}$ ones, with $\mathrm{n}=1,2,3,4,5$, and 6 , in the even-A and odd-A Sn isotopes. The only missing state is the $18^{+}$state from the $v\left(\mathrm{~h}_{11 / 2}\right)^{6}$ configuration, which is not yrast. 


\section{Conclusion}

Thanks to the high efficiency of the Euroball array, high-spin states have been identified in ${ }^{119-126} \mathrm{Sn}$ isotopes. These nuclei have been produced as fission fragments in two reactions induced by heavy ions: ${ }^{12} \mathrm{C}+{ }^{238} \mathrm{U}$ at $90 \mathrm{MeV}$ bombarding energy, ${ }^{18} \mathrm{O}+{ }^{208} \mathrm{~Pb}$ at $85 \mathrm{MeV}$. The level schemes have been built up to 5-7 $\sim \mathrm{MeV}$ excitation energy by analysing triple $\gamma$-ray coincidence data. Spin and parity values have been assigned to most of the high-spin states using $\gamma-\gamma$ angular correlation results. In addition, the use of the fission-fragment detector, SAPhIR, has allowed us to identify new isomeric states in the even-A ${ }^{120-126} \mathrm{Sn}$ isotopes. All the observed states can be described in terms of several broken neutron pairs occupying the $v\left(\mathrm{~h}_{11 / 2}\right)$ orbit. The maximum value of angular momentum available in this high- $j$ shell, i.e. for its mid-occupation and the breaking of the three pairs (seniority $v=6$ ), has been identified. This process is observed for the first time in spherical nuclei.

\section{References}

[1] ENSDF database, http://www.nndc.bnl.gov/ensdf/

[2] P.J. Daly et al., Z. Phys. A 323 (1986) 245

[3] R.H. Mayer et al., Phys. Lett. B 336 (1994) 308

[4] R. Broda et al., Phys. Rev. Lett. 68 (1992) 1671

[5] J.-A. Pinston et al., Phys. Rev. C 61 (2000) 024312

[6] C.T. Zhang et al., Phys. Rev. C 62 (2000) 057305

[7] R.L. Lozeva et al., Phys. Rev. C 77 (2008) 064313

[8] B. Fogelberg, K. Heyde and J. Sau, Nucl. Phys. A 352 (1981) 157

[9] S. Pietri et al., Phys. Rev. C 83 (2011) 044328

[10] A. Astier et al., Phys. Rev. C 85 (2012) 054316

[11] J. Simpson, Z. Phys. A 358 (1997 ) 139

[12] D.C. Radford, Nucl. Instrum. Methods A 361 (1995) 297

[13] Ch. Theisen et al., AIP Conf. Proc. Vol. 447 (1998) 143

[14] M.A.C. Hotchkis et al., Nucl. Phys. A 530 (1991) 111

[15] M.-G. Porquet et al., Acta Phys. Polonica B 27 (1996) 179

[16] E. Caurier and F. Nowacki, Acta Phys. Pol. B 30 (1999) 705

[17] K. Sieja, F. Nowacki, K. Langanke, and G. Martinez-Pinedo, Phys. Rev. C 79 (2009) 064310

[18] R.D. Lawson, Z. Phys. A 303 (1981) 51 